\title{
An integrated approach towards a public health perspective on chronic kidney disease
}

\section{Annemiek Dorgelo ${ }^{凶}$ and Tom A. J. Oostrom}

Increased awareness of chronic kidney disease among the general public is required to facilitate action to improve kidney health. An integrated approach involving mass media campaigns, primary health-care interventions and advocacy is needed to draw attention to chronic kidney disease and shift the focus from kidney failure to achieve a public health perspective on this disease.
4 there

is a broad unawareness of the importance of CKD among the general public
Chronic kidney disease (CKD) is a growing global health problem with enormous effects on individuals and society. Estimates suggest that the disease currently affects almost 100 million European individuals, and that it will become the fifth largest cause of death worldwide by $2040\left(\mathrm{REFS}^{1,2}\right)$. If nothing is done to mitigate this increase, CKD will have an incalculable health and social impact. The huge burden of CKD results not only from kidney failure but also from mild-to-moderate $\mathrm{CKD}$, which is associated with a $2-4 \times$ increased risk of cardiovascular disease and a reduction in life expectancy of 7-12 years ${ }^{3,4}$. However, a large part of this growing problem is preventable because CKD is associated with modifiable risk factors, such as diabetes mellitus, hypertension and obesity ${ }^{1,5}$. In addition, interventions in the early stages of CKD can potentially prevent or delay progression to kidney failure ${ }^{2,6}$. Given the alarming numbers of patients and the benefits of early intervention, one would expect CKD to be considered a serious public health problem. However, there is a broad unawareness of the importance of CKD among the general public.

Various qualitative studies show that if members of the general public are aware of kidney disease, their knowledge is generally limited to dialysis and transplantation ${ }^{1,2,7}$. Kidney disease is often seen as an inevitable consequence of ageing or a long-term complication that affects a small number of people, rather than a common and preventable chronic disease ${ }^{1,8,9}$. As a consequence, CKD seems to be perceived to be of limited relevance for the general public and a sense of urgency to act to prevent CKD is lacking, even among at-risk patients and some health professionals. A shift in perspective is needed to tackle the growing problem of CKD, from a disease-oriented focus on kidney failure to a public health perspective on kidney health and the prevention of CKD progression.

The public perception of kidney disease is influenced by communications and interventions at all levels, from health professionals, patients, their families and caregivers to politicians. In the past few years, our campaigns at the Dutch Kidney Foundation have mainly focused on increasing knowledge about the effect of kidney failure among the general public. However, given the importance of early intervention and disease prevention, we believe that it is time to shift our communication efforts towards CKD and kidney health.

An integrated approach is needed to raise awareness about CKD among the general public, including those who are at increased risk of kidney disease. This approach must include consideration of different levels of health literacy and different cultural backgrounds to avoid unintentionally contributing to health inequities. Therefore, a combination of strategies is needed, including mass media campaigns, interventions involving primary health care and municipal health services, and an advocacy strategy.

Mass media campaigns that include a clear call to action, such as adopting lifestyle measures to promote kidney health or measuring one's own kidney function, can increase awareness of CKD. For example, in 2006 the Dutch Kidney Foundation initiated the 'Kidney Check' campaign, which invited Dutch adults to order a free self-test to detect albuminuria. This campaign was fairly successful in increasing awareness (1.2 million people ordered the self-test) and led to the development of the 'Preventieconsult' initiative for early detection of cardiometabolic diseases.

Primary health-care providers have an important role in educating at-risk patients and the general public about CKD. To support them in this role, knowledge exchange with nephrologists and the development of educational materials could be helpful. The Dutch Kidney Foundation supports a 'Kidney Health' project that aims to improve screening and the monitoring of CKD within primary care. This project includes setting up regional networks of nephrologists, general practitioners 
a sense of urgency to act to prevent CKD is lacking and pharmacists with the support of health insurance providers. The nephrologists visit general practices to discuss patients and help to decide whether they should be treated in secondary or primary care. Although the results are not yet available, we have noticed growing awareness and support for efforts to improve CKD detection, monitoring and care among general practitioners, nurse practitioners and health insurers, even during the COVID-19 pandemic.

Word of mouth has a crucial role in raising awareness of health topics, especially among people with low health-literacy skills. Using the infrastructure of local initiatives, such as community centres, is key to educating the general public and influencing their perspective on kidney health ${ }^{10}$. CKD and kidney health should also be included in the education efforts of municipal health

\section{Box 1 | Towards a public health perspective of kidney disease}

Increased awareness of chronic kidney disease (CKD) among the general public is required to facilitate action to improve kidney health. A shift in focus from kidney failure to a public health perspective on CKD is needed to increase the perception of relevance of CKD and kidney health among the general public.

\section{Current focus on kidney failure}

- Limited perspective, and lack of awareness of CKD and kidney health

- Perceived to be of limited relevance for the general public

- Approach involving mass media mainly focusing on the effect of kidney failure, patient education and management of CKD

- Knowledge of health professionals mainly in secondary care

Towards a focus on kidney health and CKD prevention

- Increased awareness of CKD and kidney health

- Wider relevance for the general public

- Increased urgency to act to prevent CKD and progression to kidney failure

- Requires an integrated approach, for example, mass media campaigns, public health initiatives, health initiatives targeted at specific patient groups, health education and interventions within primary care, knowledge exchange between nephrologists and primary care providers and a stakeholder advocacy strategy services and other local health initiatives that aim to promote healthy lifestyles and prevent chronic diseases.

Finally, an advocacy strategy to engage relevant stakeholders such as health insurers and politicians is an important component of an integrated approach to increase awareness of kidney disease. Such an approach with interventions at different levels (BOX 1) — involving nephrology professionals, patients and their families, caregivers, and kidney-health advocacy organizations could improve awareness and understanding of CKD among the general public and lead to a sense of urgency to act on CKD and improve kidney health.

1. Vanholder, R. et al. Fighting the unbearable lightness of neglecting kidney health: the decade of the kidney. Clin. Kidney J. 14 1719-1730 (2021).

2. GBD Chronic Kidney Disease Collaboration. Global, regional, and national burden of chronic kidney disease, 1990-2017: a systematic analysis for the Global Burden of Disease Study 2017. Lancet 395 , 709-733 (2020).

3. United States Renal Data System. 2020 USRDS Annual Data Report: Epidemiology of Kidney Disease in the United States (National Institutes of Health, National Institute of Diabetes and Digestive and Kidney Diseases, 2020).)

4. Gansevoort, R. T. et al. Chronic kidney disease and cardiovascular risk: epidemiology, mechanisms, and prevention. Lancet 382, 339-352 (2013).

5. Versino, E. \& Piccoli, G. B. Chronic kidney disease: the complex history of the organization of long-term care and bioethics. why now more than ever, action is needed. Int J Environ. Res. Public Health 16, 785 (2019).

6. Gansevoort, R. T. \& de Jong, P. E. The case for using albuminuria in staging chronic kidney disease. J. Am. Soc. Nephrol. 20, 465-468 (2009).

7. Gheewala, P. A., Peterson, G. M., Zaidi, S. T. R., Jose, M. D. $\&$ Castelino, R. L. Public knowledge of chronic kidney disease evaluated using a validated questionnaire: a cross-sectional study. BMC Public Health 18, 371 (2018).

8. van Dipten, C. et al. What patients with mild-to-moderate kidney disease know, think, and feel about their disease: an in-depth interview study. J. Am. Board Fam. Med. 31, 570-577 (2018).

9. Neale, E. P., Middleton, J. \& Lambert, K. Barriers and enablers to detection and management of chronic kidney disease in primary healthcare: a systematic review. BMC Nephrol. 21 , 83 (2020).

10. Busch, M. C. M. \& Schrijvers, C. T. M. Effecten van Leefstijlinterventies Gericht op Lagere Sociaaleconomische Groepen (RIVM, 2010).

Competing interests

The authors declare no competing interests. 